\title{
La comunicación enfermera-paciente en la atención hospitalaria, caso México
}

\author{
Adela Alba-Leonel,* Guillermo Fajardo-Ortiz,**Eloísa Tixtha López, *** Joaquín Papaqui-Hernández****
}

\begin{abstract}
RESUMEN
La comunicación es ante todo un proceso fundamental en toda relación social, es el mecanismo que regula y hace posible la interacción entre las personas. En lo que respecta al área de salud, se han encontrado estudios en donde la comunicación en el hospital es deficiente, siendo el personal de enfermería el receptor de la confianza, dudas y preguntas sobre la información que el paciente recibe sobre su proceso. Derivado de esto, la Organización Mundial de la Salud en el año 2004 identificó a la comunicación como un área de riesgo dentro del hospital, determinando seis acciones básicas denominadas metas internacionales para la seguridad del paciente, siendo la segunda meta internacional mejorar la comunicación efectiva, determinándola en todo el proceso de hospitalización y promoviendo mejoras específicas en cuanto a la seguridad del paciente. Desarrollar una adecuada relación enfermera-paciente, desde que ingresa al hospital, nos permite elaborar una valoración más exhaustiva del estado de salud del paciente, identificando a primera vista las necesidades que se encuentran alteradas en ese momento, por lo que el proceso de comunicar es la base y eje de los cuidados de enfermería. Sin este proceso no se podrían planificar los cuidados.
\end{abstract}

Palabras clave: Relación enfermera-paciente, cuidados, comunicación.

\section{The nurse-patient communication in hospital care, case Mexico}

\begin{abstract}
Communication is primarily a fundamental process in all social relationships, is the mechanism that regulates and allows interaction between people. With respect to the area of health, there are studies where communication is poor in the hospital, nurses being the recipient of the trust, doubts and questions about the information that the patient receives on its process. Derived from this, the World Health Organization in the year 2004, identified communication as an area of risk within the hospital, identifying six basic actions called international goals for patient safety. As the second international goal: Improve effective communication, determining it throughout the hospitalization process, promote specific improvements in terms of patient safety. Develop adequate nurse - patient from entering hospital allows us to develop a more comprehensive assessment of the state of health of the patient, identifying the first view needs that are altered at that time, so that the process of communicating is the base and shaft of nursing care. Without this planning process could not care.
\end{abstract}

Key words: Nurse-patient relationship, care, communication.

\footnotetext{
* Lic. en Enfermería y Obstetricia, Mtra. en Ciencias en Epidemiología. Profesor de Carrera Asociado “C”, Secretaría General, Escuela Nacional de Enfermería y Obstetricia, UNAM.

** Médico Cirujano, Master of Hospital Administration, Maestría en Ciencias Administrativas y Doctor en Historia. Profesor Titular "C", Facultad de Medicina, UNAM.

*** Lic. en Enfermería y Obstetricia, Escuela Nacional de Enfermería y Obstetricia, UNAM.

***** Ing. en Informática, Área de Integración de Información, División de Información en Salud, Dirección de Prestaciones Médicas, IMSS.
}

Correspondencia: M. en C. Adela Alba-Leonel. Antiguo camino a Xochimilco y Viaducto Tlalpan, Col. San Lorenzo Huipulco, Tlalpan, 14370, México, D.F.Tel. 555623 32, ext. 239. E-mail: adelaalbaleonel@yahoo.com.mx

Este artículo puede ser consultado en versión completa en http://www.medigraphic.com/enfermerianeurologica 


\section{LA COMUNICACIÓN ENFERMERA-PACIENTE EN EL CUIDADO}

L os seres humanos tienen por naturaleza comunicarse a lo largo de la historia de la humanidad; este proceso es constante y por ende genera entre las personas una interacción que puede mantener a un individuo o a la sociedad en general en una relación mutua.

Desde las civilizaciones antiguas, la comunicación ha tenido un papel importante; por ejemplo, cada civilización tiene un código de comunicación como: señales, gestos y formas que son exclusivas para cada cultura. Tomando en cuenta lo anterior para que estas culturas siguieran bajo el mismo estatus, fue indispensable que desarrollaran un lenguaje que les permitiera comunicarse. La comunicación entonces tiende a ser una herramienta indispensable por la cual se transmite información de cualquier tipo, generando con ello un intercambio de perspectivas, experiencias y opiniones. Sin embargo, aun cuando los seres humanos tenemos la capacidad de comunicar, este proceso no es adoptado con frecuencia o las formas en las que se realizan no son del todo las adecuadas y el mensaje que se desea transmitir no es captado en su totalidad. En la actualidad, derivado de los cambios en la población, aspectos demográficos y migratorios, la comunicación se vuelve deficiente, por lo que existen evidencias de que este proceso no se realiza y que en ciertos sectores como el de la salud existe este problema. En el ambiente hospitalario es frecuente observar que todo el personal tiene un ritmo de trabajo acelerado; sin embargo, la comunicación no puede ser escasa, debe ser constante y existir una relación de compañerismo, que permita a los miembros del equipo de salud realizar sus actividades de manera que el paciente reciba de ellos atención con calidad. Si bien estos cambios en la población repercuten en la sociedad mexicana, el acceso a los servicios de salud se vuelve más demandante y el profesional de enfermería por estar el mayor tiempo con los pacientes tienen una mayor responsabilidad, ya que es común que la paciente acuda a él ante cualquier duda; sin embargo, por la carga de trabajo, falta de tiempo o por omisión no se realiza esta labor fundamental. Desde hace mucho tiempo, se ha caracterizado la relación enfermera-paciente como un "modelo de maternalismo" enfermero, que no es más que la actitud del profesional que considera al enfermo incapacitado para poder decidir sobre su propia situación de enfermedad y para la aplicación por sí mismo de los cuidados y de los procedimientos, y técnicas que le pueden ser de ayuda. ${ }^{1}$ En muchas ocasiones la actitud del profesional enfermero se ha caracterizado por no dar ninguna información al enfermo sobre lo que se le realiza, y le ha restado importancia a su situación de enfermedad con el propósito de evitarle sufrimiento, pero dejando al enfermo sin poder decidir, por desconocimiento de la realidad sobre temas que atañen a su salud.
Este modelo "maternalista de enfermería" hace que los pacientes y la familia acudan a ella ante cualquier aspecto de la enfermedad. Por tanto, resulta de importancia conocer cuál es la importancia de la comunicación para los profesionales de la salud, en especial para el profesional de enfermería, no sólo por el resultado que se genera de sus cuidados, sino también por la habilidad que desarrolla al estar en contacto con el paciente por más de ocho horas, en cada uno de los turnos en los que trabaja. La comunicación es importante ya que sin ella los seres humanos no podrían mantener una relación.

\section{LA IMPORTANCIA DE LA COMUNICACIÓN HOSPITALARIA}

La comunicación es ante todo un proceso fundamental en toda relación social; es el mecanismo que regula, hace posible la interacción entre las personas, ${ }^{2}$ y permite desarrollar un lazo afectivo que establece en el individuo la capacidad de comunicarse con otras personas, generando un patrón cíclico y continuo.

Se han encontrado estudios ${ }^{2,3}$ en donde la comunicación en el hospital es deficiente, siendo el personal de enfermería la receptora de la confianza, dudas y preguntas sobre la información que el paciente recibe sobre su proceso, ${ }^{4}$ si bien el estado emocional del paciente es distinto al momento de su internamiento y cuando la enfermera intercambia experiencias con los pacientes, se propicia un ambiente de confianza y estabiliza su estado emocional, en un ambiente tranquilo, cooperará con el tratamiento farmacológico y su estadía en el hospital será agradable contrario a lo que percibía antes de establecer una comunicación con la enfermera.

Cada paciente tiene una forma diferente de interpretar los mensajes; sin embargo, se debe establecer una relación de ayuda para que el mensaje sea entendido en su totalidad. ${ }^{5}$ Siendo la comunicación una necesidad del paciente y de la familia, al mismo tiempo permite al paciente conocer su estado de salud y estar actualizado respecto a su enfermedad. Peplau opinaba que las intervenciones de enfermería son procesos interpersonales terapéuticos y significativos para la persona. Cuidados personalizados con especial hincapié en la relación enfermera-paciente, ${ }^{2}$ para fortalecer esta relación es necesario que la enfermera desarrolle las habilidades específicas relacionadas con las patologías de los pacientes; así mismo, con ellos se mantendrá actualizada, obtendrá conocimiento y actitudes que contribuirán al desarrollo óptimo de una buena relación con el paciente. Para conseguir una buena relación es necesario que la enfermera involucre su propia experiencia, para ayudarla a transformarse en una persona genuina dentro de la interacción, y pueda ser percibida por el paciente como un profesional totalmente involucrado, ${ }^{6}$ evitando quejas y expresiones como: "ni siquiera me puso atención" o "se mostraba 
indiferente a lo que yo le transmitía"? Así mismo, la comunicación con el paciente propiciará una relación de ayuda con cuidados holísticos a cada uno de los pacientes.

En la actualidad, los servicios de salud tienen más demandas debido a la ampliación de la seguridad social, los pacientes demandan y exigen cada vez mayor información, ya que el nivel cultural de la población ha aumentado y conocen la existencia de los derechos y si no los conocen es deber del profesional de enfermería informarles, ${ }^{8}$ esta nueva generación de pacientes se encuentran más informados y por ende solicitan mayor atención e información, tal como se establece en la carta de los derechos de las y los pacientes en México. ${ }^{9}$ El personal de enfermería debe propiciar el cumplimiento de este derecho y difundirlo entre los pacientes, tal como se instituye en el Código de Ética para las Enfermeras y Enfermeros en México, ${ }^{10}$ en su Capítulo II. "De los deberes de las enfermeras para con las personas", en sus Artículos Segundo, Tercero y Sexto, donde se establece:

Respetar la vida, los derechos humanos y, por consiguiente, el derecho de la persona a decidir tratamientos y cuidados una vez informado; mantener una relación estrictamente profesional con la persona, en un ambiente de respeto mutuo y de reconocimiento de su dignidad, valores, costumbres y creencia y comunicar a la persona los riesgos, cuando existan, y los límites que tiene el secreto profesional ante circunstancias que impliquen mala intención o daño a terceros.

Por su parte, en el Código Deontológico de Enfermería en España ${ }^{11}$ en su Capítulo II: Artículos 10 y 11, establecen que: "Es responsabilidad de la Enfermera/o mantener informado al enfermo, tanto en el ejercicio libre de su profesión como cuando éste se ejerce en las instituciones sanitarias, empleando un lenguaje claro y adecuado a la capacidad de comprensión del mismo"; así mismo, "la Enfermera/o deberá informar verazmente al paciente, dentro del límite de sus atribuciones. Cuando el contenido de esa información exceda del nivel de su competencia, se remitirá al miembro del equipo de salud más adecuado". Por lo tanto, podemos dar información referente a todas las actuaciones de las cuales somos responsables, sobre lo que tenemos conocimiento, la habilidad y la destreza para su desarrollo, es decir, en las que somos competentes. El objetivo principal del proceso de informar es establecer una relación de confianza o una relación terapéutica para mejorar la atención de los familiares, siendo por tanto un deber de la enfermería. ${ }^{12}$ La comunicación es de tal importancia que al identificar las áreas de riesgo dentro del hospital en el año 2004, la Organización Mundial de la Salud ${ }^{13}$ determinó seis acciones básicas denominadas metas internacionales para la seguridad del paciente, en las que detectó áreas problemáticas y describió soluciones a los problemas, basándose en evidencias y el conocimiento de expertos. La segunda meta internacional es mejorar la comunicación efectiva, determinándola en todo el proceso de hospitalización y promoviendo mejoras específicas en cuanto a la seguridad del paciente.

\section{COMUNICACIÓN VERBAL Y NO VERBAL}

Existen diversas formas de comunicarse: expresión verbal, no verbal, visual, gestos y expresiones, así como postura y posición. A pesar de que la comunicación verbal es la que más se utiliza, la no verbal es el reflejo de lo que realmente queremos comunicar y se evidencia de distintas maneras: la forma en cómo comunicamos y si en verdad se muestra un interés en lo que se dice. Así pues, el personal de enfermería debe dejar de lado las situaciones personales y enfocarse en la situación actual del paciente, debe facilitar el diálogo, trabajar con sencillez, humanidad y humildad, liderar el equipo de trabajo, cumplir con la palabra dada, acercarse, ponerse en el lugar del otro; llamar al paciente por su nombre, respetar su intimidad y libertad de expresión. ${ }^{14}$ Es por ello por lo que debe existir una coordinación entre lo que se comunica al paciente y la manera de actuar ante la comunicación, siendo entonces una habilidad que se debe desarrollar continuamente. Un elemento fundamental para llevarlo a cabo depende en gran medida de la respuesta del paciente; del tono de voz, siendo ésta la herramienta más valiosa que se podría considerar como un arsenal terapéutico; además, es el principal vehículo para enviar un mensaje y transmitir confianza, seguridad, energía, emoción y entusiasmo al paciente. ${ }^{15}$ Por este motivo es que, en ocasiones, una sola palabra expresa muchas cosas, sensaciones; por lo que el personal de enfermería debe generar y mantener una relación con el paciente. En el mismo tenor, además del tono de la voz, la mirada en el proceso de comunicación es un elemento fundamental que puede mejorar la relación enfermera-paciente, si estos elementos no fueran acordes, sucedería lo contrario y el profesional de enfermería perdería credibilidad ante el paciente. El hecho de no mirar al paciente y desviar la mirada hacia otra parte del que escucha hace que se sienta incómodo y no entendido. ${ }^{610} \mathrm{Al}$ comunicarnos no sólo lo hacemos con un solo sentido, sino que además expresamos sentimientos y emociones cuando realizamos esta actividad.

$\mathrm{Al}$ desarrollar una adecuada relación enfermera-paciente, desde que este último ingresa al hospital, nos permite elaborar una valoración más exhaustiva de su estado de salud; identificando a primera vista las necesidades que se encuentran alteradas en ese momento. Sin este proceso no se podrían planificar los cuidados.

El personal de enfermería debe identificar oportunamente el tipo de comunicación que requiere cada paciente, de acuerdo con sus condiciones de salud como, por ejemplo, un paciente entubado que requiera de una comunicación no 
verbal: lo más importante de esta relación es mantener la comunicación según sus posibilidades de salud. Por lo anterior, la relación de enfermera-paciente debe ir más allá sin quedarse sólo en la interpretación de los signos y síntomas. También es preciso establecer una verdadera empatía, entendiendo ésta como la capacidad de reconocer y compartir emociones y estados de ánimo con la otra persona para así comprender el significado de su conducta. ${ }^{16}$ Una buena relación enfermera-paciente nos permite elaborar un mejor plan de cuidados: más personalizado, humanizado y ético, con el propósito de mejorar el estado de salud del paciente, haciendo su estancia más agradable en el hospital y reducir de manera significativa el miedo y la ansiedad que genera la hospitalización.

Así mismo, toda información que se proporcione, tanto al paciente como al familiar, debe ser entregada por escrito en forma clara y entendible.

\section{LA COMUNICACIÓN EN LA FORMACIÓN PROFESIONAL}

Para fortalecer la comunicación y tener un impacto de la relación enfermera-paciente es necesario que ésta se realice de manera constante.

Durante la formación del profesional de enfermería, él asiste a prácticas clínicas y comunitarias, donde adquiere las habilidades que requiere la profesión; sin embargo, se ha demostrado en diferentes estadios ${ }^{2,3,10}$ que la formación en comunicación es deficiente para llevar a cabo una buena relación enfermera-paciente, esta misma situación se observa para el caso de México.

\section{CONSIDERACIONES}

Los profesionales de enfermería conocen y aplican las técnicas de comunicación con los pacientes; sin embargo, cabe mencionar que estas técnicas fueron adquiridas fuera del curriculum de base de la carrera de enfermería, ya que en gran parte de los planes de estudios no incluyen la asignatura de comunicación, o bien, no está en los contenidos de los programas de la(s) asignatura(s), esto significa que en su formación hay un vacío en lo que respecta al proceso de comunicación. ${ }^{2}$ Egresan con todas las habilidades excepto la de comunicación, lo cual no les permite interaccionar y comunicarse fácilmente con el paciente. El estudio de la Escuela de Enfermería de Lérida también reconoce que al personal de enfermería le falta preparación en este campo, y señala la necesidad de que éste se brinde a través de los programas de formación y de que se incluya dentro de los planes de estudios de enfermería de las escuelas universitarias. ${ }^{2}$ Es menester incluir dentro del mapa curricular de las escuelas de enfermería, la materia de comunicación como obligatoria junto con prácticas clínicas que le permita al estudiante desarrollar una actitud más empática con el paciente. Bajo esta premisa Peplau advierte que su preocupación fundamental, en lo que concierne a una escuela de Enfermería, no es pensar primero en el paciente, sino contribuir con desarrollo gradual de cada alumno orientándolo hacia la formación de una madurez que le permita realmente "cuidar" al paciente. Para cumplir con esta premisa se requiere que los profesionales de enfermería estén preparados en esta relación enfermera-paciente. Con ello mejorará la calidad en la atención, será más holística, humanizada y personalizada. En suma, la comunicación con el paciente es la base de todos los cuidados de enfermería. ${ }^{6}$

\section{BIBLIOGRAFÍA}

1. Orbezogo AA. Ética y enfermería. Disponible en: https://www.paliativossinfronteras.com/upload/publica/.../02\%20Orbegozo.pdf [Consultado el 16 de octubre de 2012]

2. Noreña PAL, Cibanal JL, Alcaraz MN. La interacción comunicativa en el cuidado de la salud. Rev Esp Com Sal 2010; 1 (2): 113-129.

3. García MMI, Nieves LIM, Vicente EMJ. Reflexiones en torno a la Relación Terapéutica ¿Falta tiempo? Index Enferm (Gran) 2004; (47): 44-48.

4. Haro FF, Martínez LBM. Instrumentalizar la comunicación en la relación enfermera-paciente como aval de la calidad. Rev Calidad Asistencial 2002; 17 (8): 39-44.

5. Pinzón SL. El plan de alta: una herramienta para el cuidado integral y la recuperación de la persona enferma [Serie en línea]; 2005. Disponible en: https://promocionsalud.ucaldas.edu.co/dolados/revista.php [Citado el 21 de septiembre de 2012]

6. González OY. La enfermera experta y las relaciones interpersonales. Rev Aquichan Universidad de La Sabana Chia, Colombia. Octubre 2007; 7 (7): 129-138.

7. Camarena RE, Hernández TF, Fajardo DG. La comunicación humana y su relación con la queja médica. Revista CONAMED 2011; 16 (3): 141-147.

8. Función de la enfermería en la comunicación con el paciente. Proyecto de Investigación. Universidad de Vigo. Noviembre, 2008. Disponible en: https:// [Consultado el 25 de septiembre de 2012]

9. Carta de los Derechos Generales de las pacientes y los pacientes, 2001. Disponible en: http://www.conamed.gob.mx/publicaciones/pdf/derechos_pacientes.pdf [Consultado el 30 de agosto de 2012]

10. Código de Ética para las Enfermeras y Enfermeros en México, 2001. Disponible en: https://www.ssa.gob.mx/código/enfermería. México_pdf [Consultado el 30 de mayo de 2012]

11. Código Deontológico de la Enfermería Española, 2003. Disponible en: https://www.unav.es/cdb/esotcodigoenf.html [Consultado el 30 de septiembre de 2012]

12. Pérez-Fernández MC, Navarro-Infante FR, Dulce-García MA, GallardoJiménez N, Fernández-Fernández A. Comunicación: Una necesidad para el Paciente-Familia. Una competencia de Enfermería. Revista Digital de Enfermería 2009; (13): 15-20. Disponible en: http://www.paginasenferurg.com/

13. Metas Internacionales para la Seguridad del Paciente. OMS 2004. Disponible en: http://www.metas:inernacionales.sp.gob.mx.pdf [Consultada el 30 de septiembre de 2012]

14. Naranjo BIC, Ricaurte GGP. La comunicación con los pacientes. Rev Investigación y Educación en Enfermería-Medellín 2006; 24 (1): 93-98.

15. López EM, Vargas LR. La comunicación interpersonal en la relación enfermera-paciente. Rev Enferm IMSS 2002; 10 (2): 93-102.

16. Mercedes ZH. Quinta Jornada de Bioética. Relaciones interpersonales en el ámbito del cuidado. El rol distintivo de la Enfermería; 2003. Disponible en: http://www.familia.org.Jornada_05_rela_interpersonales. html [Consultada el 25 de septiembre de 2012]

17. Maya RB. Relación profesional enfermera-paciente; 2009. Disponible en: http://www.euosuna.org_relacionprofenfermerapaciente2009.doc [Consultada el 25 de septiembre de 2012] 